\title{
Retinol sérico de adolescentes de uma escola da cidade de São Paulo
}

\author{
Retinol blood levels in high school students \\ of São Paulo, Brazil
}

Márcia Regina VÍTOLO ${ }^{1}$

Cintia Mendes GAMA ${ }^{1}$

Suzana de Souza QUEIROZ²

Fábio Ancona LOPEZ $^{3}$

Fernando Antonio Barile COLUGNATI ${ }^{4}$

R E S U M O

\section{Objetivo}

Investigar os níveis séricos de retinol de 218 adolescentes de ambos os sexos com idade entre 10 e 19 anos, matriculados em colégio da rede privada de ensino da cidade de São Paulo, foi o objetivo deste trabalho.

\section{Métodos}

Para a avaliação da condição nutricional dos adolescentes, utilizaram-se as medidas antropométricas de peso e altura e também a história dietética. A dosagem de retinol foi realizada pela técnica de espectrofotometria, considerando como níveis séricos inadequados valores $<1,05 \mu \mathrm{mol} / \mathrm{L}(30 \mu \mathrm{g} / \mathrm{dL})$.

\section{Resultados}

A média de ingestão, de acordo com a faixa etária, foi maior que a recomendação, porém com altos valores de desvios padrão, em ambos os sexos. A prevalência de adolescentes com níveis séricos de vitamina $\mathrm{A}$ abaixo do adequado foi de 30\% em ambos os sexos e a análise da correlação de Índice de Massa Corporal com os níveis séricos de retinol não mostrou significância, como também a análise da correlação entre a densidade de ingestão de vitamina $\mathrm{A}$ e níveis séricos de retinol.

\footnotetext{
1 Curso de Nutrição, Centro de Ciências da Saúde, Universidade do Vale do Rio dos Sinos. Caixa Postal 551, 93022-970, São Leopoldo, RS, Brasil. Correspondência para/Correspondence to: M.R. VÍTOLO. E-mail: vitolo@bios.unisinos.br

2 Departamento de Pediatria, Faculdade de Medicina de Botucatu, Universidade Estadual Paulista. Botucatu, SP, Brasil.

3 Departamento de Pediatria, Universidade Federal de São Paulo, Escola Paulista de Medicina. São Paulo, SP, Brasil.

${ }^{4}$ Departamento de Estatística, Instituto de Matemática, Estatística e Computação Científica. Universidade Estadual de Campinas. Campinas, SP, Brasil.
} 


\section{Conclusão}

Os achados deste estudo sugerem a necessidade da realização de mais investigações sobre vitamina A no período da adolescência, para verificar se o nível sérico baixo desse nutriente é um fator de risco para a saúde do adolescente ou é reflexo da captação acelerada que ocorre para atender necessidades metabólicas.

Termos de Indexação: adolescente, retinol sérico, estado nutricional.

\section{A B S T R A C T}

\section{Objective}

The purpose of the study was to examine plasma retinol levels in 218 adolescents of both sexes, ages 10 to 19 years old. All the subjects attended a private high school in the city of São Paulo.

\section{Methods}

In order to evaluate the students' nutritional condition, the researchers performed anthropometrical measurements - weight and height - in addition to investigating the subjects' diet history. The retinol level was measured through the spectrophotometer technique, considering as inadequate values of concentration under $<1.05 \mu \mathrm{mol} / \mathrm{L}(30 \mu \mathrm{g} / \mathrm{dL})$.

\section{Results}

The results indicate that the average intake, according to the age range, was higher than recommended, even though presenting high deviation patterns for both genders. The percentage of teenagers with plasma concentrations of vitamin A under the adequate level was 30\% for both genders, and neither the analysis of the correlation between Body Mass Index and plasma concentrations of retinol, nor the analysis of the correlation between the density of vitamin A intake and plasma concentration of retinol, were significant.

\section{Conclusion}

The research findings point out to the need for further investigation of vitamin A concentrations during adolescence, in order to check more accurately whether the low plasma concentration of this nutrient should be considered a risk factor, or the result of the accelerated absorption which takes place in adolescence to fulfill the metabolic requirements characteristic of this period of human growth.

Index terms: adolescent, retinol blood level, nutritional status.

\section{N T R O D U Ç Ã O}

A vitamina A participa da regulação da secreção do hormônio de crescimento (GH) e IGF1 (Insulin Growth Factor-1), além de outras funções já bem consagradas ${ }^{1,2}$. Uma das transformações somáticas mais notáveis que ocorrem na adolescência é o aumento da velocidade de crescimento; mais precisamente nesse período o adolescente obtém $50 \%$ do peso adulto, $45 \%$ da massa esquelética e $25 \%$ da estatura final. $O$ estado nutricional relacionado à vitamina $A$, durante essa fase, pode interferir nos processos do crescimento e da maturação sexual, os quais estão intensificados no período do estirão pubertário ${ }^{3,4}$.

A ingestão adequada de vitamina A está atrelada à favorável condição socioeconômica e, independente desse fator, ao hábito alimentar individual ou familiar que por diversos fatores culturais e ambientais promovem o consumo de alimentos com maior concentração desse nutriente. O consumo de vitamina A pela 
população, apresenta distribuição muito heterogênea, resultando em um coeficiente de variação maior do que 100 para a maioria dos grupos populacionais estudados, de acordo com os dados publicados pelas novas recomendações nutricionais ${ }^{5}$, o que implica em limitações aos resultados obtidos a partir da estimativa do retinol em inquéritos alimentares. Apesar das limitações dessa análise, considerou-se as bases de dados das recomendações nutricionais, os inquéritos alimentares e as tabelas de composição dos alimentos disponíveis, para um estudo realizado com 724 adolescentes, provenientes de duas escolas (particular e pública) da vila Mariana da cidade de São Paulo; a pesquisa revelou que 64\% dos jovens consumiam apenas $20 \%$ das quantidades de vitamina $A$ recomendadas para a faixa etária ${ }^{6}$. Os níveis séricos de retinol não refletem os depósitos hepáticos desse nutriente, mas podem ser aceitos como expressão da condição adequada do estado nutricional relacionado com a vitamina $\mathrm{A}$ ou como indicativo de risco para deficiência da vitamina ${ }^{7}$.

Diante dessas considerações, o presente estudo, investigou os adolescentes matriculados em escola particular da cidade de São Paulo, com o propósito de se conhecer a situação do estado nutricional relacionado com a vitamina A entre adolescentes de bom nível socioeconômico, já que existem poucos estudos em nosso meio que tenham realizado essas determinações nesse grupo etário ${ }^{8,9,10}$.

\section{CASUÍSTICA E MÉTODOS}

A amostragem foi constituída por uma sub-amostra de 218 adolescentes de ambos os sexos, sendo 91 do sexo feminino $(41,7 \%)$ e 127 do sexo masculino (58,3\%), entre 418 adolescentes de uma escola particular localizada na região urbana da cidade de São Paulo. Estes foram selecionados aleatoriamente por meio de um programa de sorteio elaborado segundo os padrões da Disciplina de Estatística do
Departamento de Medicina Preventiva da Universidade Federal de São Paulo. O total de adolescentes matriculados na escola no período estudado era de 2 822. A presente investigação não incluiu toda a amostra selecionada pois a coleta de sangue já tinha sido iniciada para outras análises, quando se obteve a oportunidade de determinar os níveis séricos de retinol. Desse modo, todos os procedimentos das técnicas necessárias para a coleta de sangue, considerando a análise específica de vitamina A, foram realizados a partir de novos agendamentos. Os adolescentes foram convidados a participar da investigação e, após o aceite, seus pais receberam carta de autorização para que assinassem se estivessem de acordo com a realização da pesquisa. A partir de então, os dados relativos ao inquérito alimentar e à avaliação antropométrica foram coletados por nutricionista. O protocolo de pesquisa foi aprovado pela Comissão de Ética do Conselho de Pós-Graduação e Pesquisa da Universidade Federal de São Paulo, Escola Paulista de Medicina.

Para a coleta de informação sobre a ingestão de vitamina A foi utilizado o método de registro da história dietética ${ }^{11}$. Adotou-se como ponto de corte a freqüência da ingestão maior ou igual a quatro vezes na semana, para exprimir consumo que pudesse ser considerado adequado ao estado nutricional dos indivíduos.

Para realização do cálculo da dieta foi utilizado o Sistema de Apoio à Nutrição Versão 2,5 do Centro de Informática à Saúde da Universidade Federal de São Paulo, Escola Paulista de Medicina ${ }^{12}$ e tabelas de composição química dos alimentos ${ }^{13,14}$ além das informações obtidas das indústrias de alimentos sobre produtos não referenciados nas tabelas. Para avaliação da densidade do nutriente, determinou-se a proporção do consumo de vitamina A por mil calorias $^{15}$.

Os adolescentes foram pesados e medidos no momento da entrevista e realizou-se a anamnese alimentar. A avaliação do estado nutricional foi feita a partir do Índice de Massa Corporal (IMC), utilizando-se como referencial de 
percentis a tabela de Must et al. ${ }^{16}$. Os pontos de corte para classificação de baixo peso, eutrofia e excesso de peso, foram: $<5^{\text {th }}$, entre $5^{\text {th }} \mathrm{e}<85^{\text {th }} \mathrm{e}$ $\geq 85^{\text {th }}$, respectivamente, conforme as recomendações da Organização Mundial de Saúde ${ }^{17}$.

Um total de $1 \mathrm{~mL}$ de sangue foi coletado via venosa, colocado em tubo tipo Eppendorf e imediatamente centrifugado em microcentrífuga. O soro foi separado com auxílio de seringa com agulha, ainda no local da coleta e, com a agulha, perfurou-se a tampa de tubo vacuotainer, de maneira a se evitar exposição ao oxigênio. O tubo foi devidamente rotulado e envolto em papel preto. As etapas de coleta de sangue, centrifugação e separação do soro foram efetuadas em ambiente protegido da ação solar, na ausência de luz direta. Os soros foram armazenados em freezer a $-20^{\circ} \mathrm{C}$ e transportados em isopor, com gelo seco, para o Laboratório de Pesquisa do Departamento de Pediatria da Faculdade de Medicina de Botucatu, onde foram efetuadas dosagens séricas de vitamina $A$. A vitamina $A$ foi dosada pelo método de Bessey-Lowrey ${ }^{18}$, modificado por Araújo \& Flores ${ }^{19}$.

Os valores utilizados para classificação de deficiência sérica de vitamina A foram os seguintes:

$$
\begin{aligned}
& <0,35 \mu \mathrm{mol} / \mathrm{L}(<10 \mu \mathrm{g} / \mathrm{dL})=\text { deficiência } \\
& \text { grave } \\
& 0,35 \mu \mathrm{mol} / \mathrm{L}-<0,70 \mu \mathrm{mol} / \mathrm{L} \\
& (10-<20 \mu \mathrm{g} / \mathrm{dL})=\text { deficiência moderada } \\
& 0,70 \mu \mathrm{mol} / \mathrm{L}-<1,05 \mu \mathrm{mol} / \mathrm{L} \\
& (20-<30 \mu \mathrm{g} / \mathrm{dL})=\text { deficiência leve }
\end{aligned}
$$

Propõe-se que os níveis menores de $1,05 \mu \mathrm{mol} / \mathrm{L}(<30 \mu \mathrm{g} / \mathrm{dL})$ também sejam considerados como sugestão de depleção crítica das reservas de vitamina $A$. Os valores considerados adequados, portanto, são aqueles iguais ou acima de $1,05 \mu \mathrm{mol} / \mathrm{L}(\geq 30 \mu \mathrm{g} / \mathrm{dL})^{20}$.

Para comparar os níveis séricos de vitamina A entre os sexos e as faixas etárias foi utilizado o teste " $\mathrm{t}$ " de Student e o teste quiquadrado para a ingestão dietética desse nutriente. Para analisar a existência de correlação entre os níveis séricos de vitamina A com IMC e a densidade do nutriente, utilizou-se o teste de correlação de Spearman ${ }^{21}$. Fixou-se $p<0,05$ como nível de significância, assinalado com asterisco (*).

\section{RES U LT A D O S}

A população estudada apresenta média de idade em anos de $12,3 \pm 2,2$ e 13,2 $\pm 2,2$ para 0 sexo feminino e masculino, respectivamente. 0 valor médio do índice utilizado para classificação do estado nutricional foi de $19,1 \mathrm{~kg} / \mathrm{m}^{2} \pm 3,1 \mathrm{~kg} / \mathrm{m}^{2}$ e $20,4 \mathrm{~kg} / \mathrm{m}^{2} \pm 3,8 \mathrm{~kg} / \mathrm{m}^{2}$, de acordo com a mesma ordem de sexo (Tabela 1).

Verificou-se que $16,5 \%$ do sexo feminino e $22,8 \%$ do sexo masculino apresentaram valores de retinol sérico abaixo de $1,05 \mu \mathrm{mol} / \mathrm{L}$ e acima de $0,70 \mu \mathrm{mol} / \mathrm{L}$, o que permite considerá-los como indivíduos com risco para depleção de depósitos; a proporção de adolescentes com deficiência

\begin{tabular}{|c|c|c|}
\hline \multirow{2}{*}{ Variáveis } & Feminino & Masculino \\
\hline & Média \pm DP & Média \pm DP \\
\hline Idade (anos) & $12,3 \pm 2,2$ & $13,2 \pm 2,3$ \\
\hline Estatura $(\mathrm{cm})$ & $151,7 \pm 9,0$ & $158,8 \pm 13,5$ \\
\hline Peso (kg) & $44,4 \pm 10,2$ & $51,6 \pm 13,9$ \\
\hline IMC (kg/m²) & $19,1 \pm 3,1$ & $20,4 \pm 3,8$ \\
\hline
\end{tabular}
moderada foi de $12,1 \%$ para o sexo feminino e 5,5\% para o sexo masculino; dos 218 adolescentes avaliados, quatro apresentaram valores menores

Tabela 1. Caracterização geral dos adolescentes quanto ao valor médio e desvio-padrão ( $\pm D P)$ em relação à idade, estatura, ao peso e ao índice de massa corporal (IMC), segundo sexo. 
que $0,35 \mu \mathrm{mol} / \mathrm{L}$, sendo três do sexo feminino (Tabela 2). Os valores médios de retinol sérico foram $1,37 \mu \mathrm{mol} / \mathrm{L}$ e $1,40 \mu \mathrm{mol} / \mathrm{L}$ para sexo feminino e masculino, respectivamente, não havendo diferença estatística entre eles $(p=0,29)$ (Tabela 3 ). Em relação às faixas etárias, valores de $1,38 \mu \mathrm{mol} / \mathrm{L} \pm 0,59 \mu \mathrm{mol} / \mathrm{L}$ foram encontrados para as adolescentes de 10 a 13 anos e valores de $1,32 \mu \mathrm{mol} / \mathrm{L} \pm 0,61 \mu \mathrm{mol} / \mathrm{L}$ para a faixa etária maior; não houve diferença significante $(p=0,74)$ entre esses valores; os valores correspondentes, para o sexo masculino foram $1,33 \mu \mathrm{mol} / \mathrm{L} \pm 0,48 \mathrm{e}$ $1,56 \mu \mathrm{mol} / \mathrm{L} \pm 0,59 \mu \mathrm{mol} / \mathrm{L}$; valores maiores foram observados na faixa etária, de 14 a 19 anos $(p=0,01)$.

A avaliação da ingestão alimentar mostrou valores compatíveis com o esperado para adolescentes provenientes de padrão socioeconômico privilegiado, no que diz respeito à ingestão energética e de macronutrientes. Os resultados da ingestão de vitamina A mostraram valores medianos de 540,0 $\mu \mathrm{g}$ EqRe para o sexo feminino e de $673,6 \mathrm{mgEqRe}$ para o sexo masculino (Tabela 3), e os valores médios foram de $814,2 \pm 777,8 \mu \mathrm{gEqRe}$ e 1009,5 $\pm 1105,0 \mu \mathrm{gEqRe}$ na mesma ordem de sexo. As recomendações atuais sugerem que se utilize a Estimated Average Requirement (EAR) para avaliar o consumo de grupos populacionais ${ }^{22}$. Os valores de EAR para meninas de 9 a13 e de 14 a 18 anos são de $420 \mu \mathrm{gEqRe}$ e $630 \mu \mathrm{gEqRe}$, respectivamente. Para os meninos das mesmas faixas etárias os valores

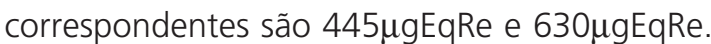
Assim, as análises de acordo com as faixa etárias mostraram valores médios de $851 \pm 823 \mu \mathrm{gEqRe}$ para as adolescentes de menor idade, com mediana de $541 \mu \mathrm{gEqRe}$; valor médio de $652 \pm 524 \mu \mathrm{gEqRe}$ para as de faixa etária maior, com mediana de $452 \mu \mathrm{gEqRe}$. Os meninos de 9 a 13 anos apresentaram a maior média de consumo

Tabela 2. Distribuição dos níveis séricos de retinol de adolescentes de acordo com concentração sérica e sexo.

\begin{tabular}{|c|c|c|c|c|c|c|}
\hline \multirow{2}{*}{ Vitamina A (sérica) } & \multicolumn{2}{|c|}{ Feminino } & \multicolumn{2}{|c|}{ Masculino } & \multicolumn{2}{|c|}{ Total } \\
\hline & $n$ & $\%$ & $\mathrm{n}$ & $\%$ & $\mathrm{n}$ & $\%$ \\
\hline$\geq 1,05 \mu \mathrm{gmol} / \mathrm{L}$ & 62 & 68,1 & 90 & 70,9 & 152 & 69,7 \\
\hline$\geq 0,70 \quad-<1,05 \mu \mathrm{gmol} / \mathrm{L}$ & 15 & 16,5 & 29 & 22,8 & 44 & 20,2 \\
\hline$\geq 0,53-<0,70 \mu \mathrm{gmol} / \mathrm{L}$ & 11 & 12,1 & 7 & 5,5 & 18 & 8,3 \\
\hline$<0,35 \mu \mathrm{gmol} / \mathrm{L}$ & 3 & 3,3 & 1 & 0,8 & 4 & 1,8 \\
\hline Total & 91 & 100,0 & 127 & 100,0 & 218 & 100,0 \\
\hline
\end{tabular}

Tabela 3. Valores médio e desvio-padrão $( \pm D P)$ de ingestão de energia (VET), percentual de macronutrientes e de vitamina A e vitamina A sérica de adolescentes, segundo sexo.

\begin{tabular}{|c|c|c|c|c|}
\hline \multirow{2}{*}{ Variáveis } & \multicolumn{2}{|c|}{ Feminino } & \multicolumn{2}{|c|}{ Masculino } \\
\hline & Média & $\pm \mathrm{DP}$ & Média & $\pm \mathrm{DP}$ \\
\hline \multicolumn{5}{|l|}{ Dieta } \\
\hline VET (kcal) & $2464,57=$ & $\pm 868,94$ & 3191,73 & $\pm 1075,23$ \\
\hline Carboidrato \% & $50,21=$ & $\pm \quad 8,30$ & 48,40 & 8,54 \\
\hline Lipídio \% & $32,68=$ & $\pm \quad 6,33$ & 33,52 & 7,62 \\
\hline Proteína \% & $17,00=$ & $\pm \quad 5,12$ & 18,39 & 5,10 \\
\hline Vitamina A ( $\mu \mathrm{gEgRe})$ & 814,22 & $\pm 777,81$ & 1009,54 & $\pm 1105,03$ \\
\hline \multicolumn{5}{|l|}{ Soro } \\
\hline Vitamina A ( $\mu \mathrm{gmol} / \mathrm{L})$ & $1,37=$ & $\pm \quad 0,59$ & 1,40 & 0,53 \\
\hline
\end{tabular}


$1094 \pm 1241 \mu \mathrm{gEqRe}$ e mediana de $695 \mu \mathrm{gEqRe}$. Para o sexo masculino da maior faixa etária o valor médio foi de $844 \pm 811 \mu \mathrm{gEqRe}$ e mediana

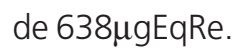

Quanto à avaliação do estado nutricional, verificou-se que $69 \%$ dos meninos eram eutróficos, 24\% tinham sobrepeso ou eram obesos e $7 \%$ estavam com o peso abaixo do esperado. Nas meninas, as porcentagens correspondentes foram $79 \%, 15 \%$ e $6 \%$ (Tabela 1 ).

A correlação entre os níveis séricos de vitamina $A$ e IMC não mostrou resultados significantes para o sexo masculino $(p=0,20$; $r=0,11)$, como tampouco para o sexo feminino $(p=0,05 ; r=0,20)$. A análise de correlação entre a densidade de ingestão de vitamina $A$ por 1000kcal e os níveis séricos de retinol não mostrou resultado significante para nenhum dos sexos. (feminino $-p=0,62 ; r=0,05$ e masculino $-p=0,60$; $r=0,04)$.

\section{DISCUSS Ã O}

Os resultados deste estudo mostraram que foi possível detectar valores séricos inadequados de retinol $(<1,05 \mu \mathrm{mol} / \mathrm{L})$ em adolescentes de boa condição socioeconômica. Para o diagnóstico correto de deficiência de vitamina A é necessária a associação entre indicadores clínicos, exame histológico, dados fisiológicos e bioquímicos. As manifestações clínicas são a expressão tardia da deficiência, sendo sua ocorrência rara nos países da America Latina e Caribe ${ }^{23}$. Por ser uma vitamina de depósito, é difícil encontrar associação direta entre o consumo alimentar no momento do estudo e a condição nutricional desse nutriente. O retinol sérico como indicador bioquímico é muito utilizado para se obter o perfil nutricional de populações em estudos epidemiológicos, além de permitir a avaliação das medidas de intervenção, tornando-se um excelente instrumento para monitorá-las ${ }^{24}$. A Organização Mundial da Saúde (OMS) estabeleceu pontos de corte dos níveis séricos de vitamina $A$, visando as estimativas do risco relativo e da prevalência, com enfoque na gravidade da deficiência populacional e na magnitude da hipovitaminose A. O ponto de corte, atualmente recomendado pela Organização Mundial da Saúde (OMS) para detectar a hipovitaminose A como sendo problema de magnitude de saúde pública, é 0,70 4 mol/L; abaixo deste nível, deve-se indicar a percentagem de indivíduos que apresentam valores indicativos de deficiência de retinol sérico. Esta carência é considerada grave quando atinge $20 \%$ ou mais da população; moderada quando atinge no mínimo $10 \%$ da população, e leve se afeta menos de $10 \%$ da população ${ }^{24}$. Os resultados deste estudo não são de base populacional, mas indicam que entre os adolescentes investigados, 10\% apresentaram valores abaixo de 0,70 $\mathrm{mmol} / \mathrm{L}$ e $30 \%$ abaixo de $1,05 \mu \mathrm{mol} / \mathrm{L}$. O parâmetro bioquímico é reconhecido pela OMS e por especialistas da área, como sendo um indicador de deficiência de vitamina $A$. Os dados encontrados neste estudo surpreendem pela posição economicamente privilegiada em que se encontram esses jovens. Em nosso meio, estudo de prevalência realizado em sete municípios do Estado de São Paulo, demonstrou que 30\% das crianças usuárias da rede básica de saúde, com idades de 6 e 23 meses apresentaram-se com níveis séricos de retinol abaixo de 0,70 $\mu \mathrm{mol} / \mathrm{L}$ e $7 \%$ abaixo de $0,35 \mu \mathrm{mol} / \mathrm{L}^{25}$. Estudo publicado em 1981, com dados populacionais colhidos entre 1969 e 1973, em comunidades provenientes de regiões menos desenvolvidas do estado de São Paulo, mostrou freqüência de $19 \%$ de adolescentes da faixa etária de 11 a 14 anos com níveis séricos abaixo de $0,70 \mu \mathrm{mol} / \mathrm{L}$. Tanto o tempo decorrido desde a realização do referido estudo, como as suas características metodológicas ${ }^{8}$ limitam a comparação com os resultados do presente trabalho.

Estudo recente avaliando níveis séricos de retinol em 285 adolescentes americanos de 12 a 17 anos revelou valores médios de 1,57 $\mathrm{mmol} / \mathrm{L}$ e $1,50 \mu \mathrm{mol} / \mathrm{L}$ em meninos e meninas respectivamente ${ }^{26}$. Tais resultados são mais elevados do que os valores médios encontrados no presente estudo 
que foram de 1,40 $\mu \mathrm{mol} / \mathrm{L}$ para o sexo masculino e $1,38 \mu \mathrm{mol} / \mathrm{L}$ para o sexo feminino. Entretanto, a idade mínima da faixa etária utilizada pode ser a justificativa para essa diferença, já que, em estudo de nível nacional de adolescentes americanos (NHANES III), os participantes tinham entre $9 \mathrm{e}$ 13 anos de idade e os valores de retinol sérico

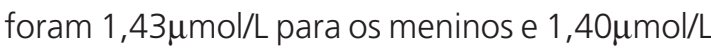
para as meninas; mais próximos, portanto, dos valores aqui encontrados, correspondentes as crianças cuja idade mínima era de 10 anos. Segundo Lewis et al. ${ }^{27}$, há alterações dos níveis séricos de retinol de acordo com a idade, isto é, valores mais elevados são observados em crianças com mais idade.

Os resultados referentes ao sexo feminino necessitam de mais investigações, pois de acordo com Brabin \& Brabin ${ }^{4}$ o período hormornal dos ciclos menstruais interfere nos níveis séricos de vitamina A. Assim, seria necessária a obtenção de dados quanto ao ciclo menstrual no dia de coleta, o que não foi realizado em nosso estudo.

Segundo Olson ${ }^{28}$ a vitamina A é rapidamente reciclada entre o fígado e outros tecidos e as modificações dos níveis de ingestão, em curto prazo de tempo, confundem a resposta quanto ao estado nutricional desse nutriente. Assim, a tentativa de correlacionar ingestão de vitamina $\mathrm{A}$ e níveis séricos desse nutriente não é conclusiva, já que a obtenção de dados dietéticos implica em várias limitações relacionadas ao tipo de inquérito, fidelidade das respostas e variação quanto aos alimentos consumidos. Estudos populacionais de consumo de vitamina A revelam valores elevados de desvio-padrão e coeficiente de variação. Os resultados apresentados pelo Institute of Medicine mostram coeficientes de variação maiores que $100 \%$ para o grupo de adolescentes. O próprio órgão não recomenda a construção da curva de probabilidade de risco de deficiência para o grupo avaliado, já que variabilidade maior que $60 \%$ a $70 \%$ indica que a ingestão diária não apresenta distribuição normal ${ }^{5}$. Segundo Lee \& Nieman ${ }^{29}$ seriam necessários 400 dias de avaliação dietética para estimar com mais precisão a ingestão de vitamina A. Adicionalmente, a vitamina A é um nutriente de depósito, de maneira que o estado nutricional relacionado com a vitamina reflete longos períodos de ingestão alimentar. Os fatores mencionados tornam difíceis o diagnóstico de deficiência ou adequação da vitamina A. Assim, como não foi possível realizar vários registros alimentares, optamos pelo método da história dietética, que permite associar o consumo quantitativo com o qualitativo.

A correlação entre IMC e vitamina A sérica foi investigada por Herberth et al. ${ }^{3}$ em adolescentes franceses de 10 a 15 anos; estes autores verificaram que a concentração de retinol apresentava correlação positiva com a gordura corporal. Tal corelação não foi confirmado no presente estudo. Naquele trabalho, ainda, foram observados, para o sexo masculino, valores de retinol sérico mais elevados do que em nossa casuística.

\section{CONCLUSÃO}

O presente estudo mostrou que um terço dos adolescentes avaliados, de bom nível sócioeconômico, apresentou valores séricos considerados de risco para deficiência de vitamina $A$. Quanto ao consumo dietético da vitamina A, foram observados valores médios maiores que a recomendação vigente para as faixas etárias correspondentes e a correlação entre a densidade de ingestão de vitamina $A$ e níveis séricos de retinol não foi significante. Nesse sentido, os achados deste estudo indicam a necessidade de mais investigações sobre a vitamina A no período da adolescência, para se verificar se o nível sérico baixo desse nutriente é um fator de risco de deficiência vitamínica ou reflexo da captação acelerada que ocorre para atender necessidades metabólicas.

\section{REFER Ê NCIAS}

1. West Jr KP. Dietary vitamin A deficiency: effects on growth, infection, and mortality. Food Nutr Bull 1991; 13:119-31. 
2. Evaion-Brior D, Porquet $D$, Therrond P, Paulsen AF, Grenede MO, François L, et al. Vitamin A and nocturnal growth hormone secretion in short children. Lancet 1994; 343:87-8.

3. Herberth B, Spyckerelle Y, Deschamps JP. Determinants of plasma retinol b-carotenoid and a-tocopherol during adolescence. Am J Clin Nutr 1991; 54:884-9.

4. Brabin L, Brabin B. The cost successful adolescent growth and development in girls in relation to iron and vitamin A status. Am J Clin Nutr 1992; 55(5):955-8.

5. Institute of Medicine National. Dietary assessment. Washington, DC: The Nacional Academy Press; 2000.

6. Gama CM. Consumo alimentar e estado nutricional de adolescentes matriculados na rede de ensino particular e pública do bairro de Vila Mariana da cidade de São Paulo, São Paulo [tese]. São Paulo: Pós-Graduação em Nutrição, Universidade Federal de São Paulo, Escola Paulista de Medicina; 1999.

7. Batista Filho M. Alimentação, nutrição e saúde. In: Rouquayrol MZ, Almeida Filho N. Epidemiologia e Saúde. 5.ed. São Paulo: MEDSI; 1999. p.353-74.

8. Roncada MJ, Wilson D, Mazzilli RN, Gandra YR. Hipovitaminose A em comunidades do estado de São Paulo, Brasil. Rev Saude Publica 1981; 15:338-49.

9. Wilson D, Silva Nery ME. Hypovitaminosis A in Rio Grande do Sul, Brazil. Preliminary study. Int J Vitam Res Suppl 1983; 24:35-44

10. Flores H, Araújo CR. Liver levels of retinol in unselect necropsy specimens: a prevalence survey of vitamin A deficiency in Recife, Brazil. Am J Clin Nutr 1984; 40(1):146-52.

11. Thompson FE, Byers T. Dietary assessment resource manual. J Nutr 1994; 124 Suppl 11:2245S-301S.

12. Anção MS, Cuppari L, Tudisco ES, Draibe AS, Sigulen DM. Sistema de Apoio à Decisão em Nutrição, Versão 2.5. Centro de Informática em Saúde da Universidade Federal de São Paulo, Escola Paulista de Medicina. São Paulo; 1993.
13. Instituto Brasileiro de Geografia e Estatísitca. Secretaria de Planejamento da República. Estudo Nacional de Despesa Familiar (ENDEF): tabela de composição de alimentos. 4.ed. Rio de Janeiro: DEDIT/CDDI; 1996.

14. Pinheiro AB, Lacerda EMA, Benzercry E, Gomes MCS, Costa VM. Tabela para avaliação de consumo alimentar em medidas caseiras. 3.ed. Rio de Janeiro: Produção Independente; 1996.

15. Hansen RG, Wyse BW. Expression of nutrient allowances per 1000 kilocalories. J Am Diet Assoc 1980; 76(3):233-27.

16. Must A, Dallal GE, Dietz WH. Reference data for obesity: 85th and 95th percentiles of body mass index (wt/ht² - a correction. Am J Clin Nutr 1991; 54:773.

17. World Health Organization. Physical status: the use and interpretation of anthropometry. Geneva: World Health Organization; 1995. Technical Report Series n.854.

18. Bessey O, Lowry OH, Brock MJ, Lopez JA. The determination of vitamin $A$ and carotene in small quantities of blood serum. J Biol Chem 1946; 11:177-88

19. Araújo CRC, Flores H. Improved spectrophotometric vitamin A assay. Letters Clin Chem 1978; 24(2): 386.

20. Flores $\mathrm{H}$. Importance of the early diagnosis of vitamin A deficiency at the epidemiological level. Int J Vitam Res 1983; 24:23-4.

21. Siegel S, Castellan Jr NJ. Nonparametrics Statistics. New York: McGraw-Hill; 1988.

22. Institute of Medicine. Dietary reference intakes. Application in Dietary planning. Washington, DC: The National Academic Press: 2003; 2003. 237p.

23. Underwood BA, Arthur A. The contribuition of vitamin A to public health. FASEB J 1996; 10:1040-48.

24. Sommer A. Vitamin A deficiency and its consequences: a fiel guide to detection and control - epidemiology. Geneva: World Health Organization; 1995. p.1-73. 
25. Souza Queiroz S, Sato K, Torres MAA. Detection of the prevalence of hipovitaminose $A$ in children under 2 years of age enrolled in Basic Health care units cities of State of São Paulo. In: 16th Proceedings of the Congress of Nutrition; 1998, Montréal. Montréal; 1998. p.292. (PT 659).

26. Neuhouser $M L$, Rock $C L$, Eldrige $A L$, Kristal $A R$, Patterson RE, Cooper DA, et al. Serum concentration of retinol, a-tocopherol and the carotenoids are influenced by diet, race and obesity in a sample of healthy adolescents. J Nutr 2001; 131:2184-91.
27. Lewis CJ, Mc Dowell MA, Sempos CT, Lewis KC, Yetley EA. Relationship between age and serum vitamin $A$ in children age 4-11. Am J Clin Nutr 1990; 52(2):353-60.

28. OIson JA. Atwater Lecture. The irresistible fascination of carotenoids and vitamin A. Am J Clin Nutr 1993; 57(6):833-9.

29. Lee RD, Nieman DC. Nutritional Assessment Anthropometric, Biochemical, Clinical, Dietary. St Louis: MOSBY; 1995.

Recebido para publicação em 11 de abril de 2001 e aceito em 20 de agosto de 2003. 